\title{
Critical Current 0- $\pi$ Transition in Designed Josephson Quantum Dot Junctions
}

\author{
H. Ingerslev Jørgensen, ${ }^{1},{ }^{\circledast}$ T. Novotný, ${ }^{2,1}$ K. Grove-Rasmussen, ${ }^{1}$ K. Flensberg, ${ }^{1}$ and P. E. Lindelof ${ }^{1}$ \\ ${ }^{1}$ Nano-Science Center, Niels Bohr Institute, University of Copenhagen, \\ Universitetsparken 5, DK-2100 Copenhagen Ø, Denmark \\ ${ }^{2}$ Department of Condensed Matter Physics, Faculty of Mathematics and Physics, \\ Charles University, Ke Karlovu 5, 12116 Prague, Czech Republic
}

(Dated: November 1, 2018)

\begin{abstract}
We report on quantum dot based Josephson junctions designed specifically for measuring the supercurrent. From high-accuracy fitting of the current-voltage characteristics we determine the full magnitude of the supercurrent (critical current). Strong gate modulation of the critical current is observed through several consecutive Coulomb blockade oscillations. The critical current crosses zero close to, but not at, resonance due to the so-called $0-\pi$ transition in agreement with a simple theoretical model.
\end{abstract}

Formation of a quantum dot (QD) between two superconductors, called a Josephson QD junction, enables study of supercurrent through a single energy level of an artificial atom (the QD). Supercurrent 1, 2, 3 through a QD 4, 5, 6, 7, 8 is a dissipationless current which flows only when a constant phase difference across the QD is maintained [9, 10]. Uncontrolled fluctuations in the phase has so far prevented observation of the full magnitude of the supercurrent (the critical current). Here we analyze Josephson QD junctions, created in a single wall carbon nanotube, with a carefully designed onchip circuit that controls phase fluctuations and thereby allows extraction of the critical current. The analysis reveals a reversal of the critical current for every electron added to the QD, due to the so-called $0-\pi$ transition 11, 12, 13, 14, 15, 16, 17, 18, 19, in agreement with a simple theoretical model. The realization of a well controlled nano-scale Josephson junction opens new routes toward superconducting quantum bits [20, 21, 22].

When a Josephson QD junction is placed in an electromagnetic environment the phase difference across the QD $(\phi)$ becomes a dynamical variable, which determines the time-averaged current, $I$, and voltage drop, $V_{\mathrm{J}}=\langle(\hbar / 2 e) d \phi / d t\rangle$, across it. The $I-V_{\mathrm{J}}$ characteristics of the junction thus depends in a non-linear fashion on the phase dynamics induced by the electromagnetic environment. Inspired by Refs. [4, 10, we utilize a designed external circuit in order to control the phase fluctuations which enables us to infer the true magnitude of the critical current, $I_{\mathrm{c}}$, from the measurable critical current/switching current, $I_{\mathrm{m}}$, by a theoretical fitting procedure. $I_{\mathrm{m}}$ can significantly differ from $I_{\mathrm{c}}$ as demonstrated previously for single wall carbon nanotube (SWCNT) based Josephson junctions [4, 5, 7.

To be able to design the external circuit we model our sample (Fig. 1(a) and (b)) by an extended resistive and capacitively shunted junction (RCSJ) model 4, yielding the schematic circuit diagram shown in Fig. 1(c). The real Josephson QD junction is represented by an ideal Josephson junction with current-phase relation $I_{\mathrm{J}}(\phi)$, in parallel with a junction capacitor $C_{\mathrm{J}}$, and junction resistor $R_{\mathrm{J}}$ accounting for current carried by multiple Andreev reflections. The resistor $R_{\mathrm{J}}$ generally depends on $V_{\mathrm{J}}$ and gate voltage $\left(V_{\text {gate }}\right)$ but for small enough voltage as used in our analysis $V_{\mathrm{J}} \ll \Delta / e$, where $\Delta \sim 0.1 \mathrm{meV}$ is the superconducting energy gap, we approximate $R_{\mathrm{J}}$ to depend only on $V_{\text {gate }}$. Assuming a sinusoidal currentphase relation $\left(I_{\mathrm{J}}(\phi)=I_{\mathrm{c}} \sin \phi\right)$ the dynamics of $\phi$ in the circuit of Fig. 1(c) becomes equivalent to the damped motion of a fictitious particle in the so-called tilted washboard potential [23]. The damping of the motion of this particle is characterized by the quality factor [4, 10.

$$
Q=\frac{\sqrt{\hbar\left[C\left(1+R / R_{\mathrm{J}}\right)+C_{\mathrm{J}}\right] /\left(2 e I_{\mathrm{c}}\right)}}{R C+\hbar /\left(2 e I_{\mathrm{c}} R_{\mathrm{J}}\right)}
$$

given by the ratio of the local minimum oscillation frequency to the friction coefficient. Low $Q$ implies higher probability of trapping the particle in a potential minimum resulting in a constant phase difference and, thus, the observation of the supercurrent.

We have used Eq. (1) to design overdamped samples shown in Fig. 1(b). The superconducting electrodes (blue leads in Fig. 11(b)) are made with a small area to reduce the junction capacitance, $C_{\mathrm{J}} \sim 1 \mathrm{fF}$. Yellow leads, connecting the superconducting electrodes to contact pads, are long thin normal metal wires designed to have a large resistance, measured to be $R \sim 1.5 \mathrm{k} \Omega$. The contact pads are fabricated with a large area to increase their capacitance, $C \sim 2 \mathrm{pF}$. By inserting the above mentioned values and $R_{\mathrm{J}} \geq h / e^{2}$ (see Fig. 3(a)), into Eq. (1) we get a strong damping $Q<1$ for $I_{\mathrm{c}} \geq 0.1 \mathrm{nA}$ which is also the smallest critical current we have been able to measure (see Fig. 4 (b)).

The measurements are performed in a ${ }^{3} \mathrm{He}^{4}{ }^{4} \mathrm{He}$ dilution fridge at a base electron temperature of $75 \mathrm{mK}$. In Fig. 1(d) we show a current biased four-probe measurement of an $I-V_{\mathrm{J}}$ curve at very low bias voltage $V_{\text {sd }} \ll \Delta / e$. In this measurement, $I_{\mathrm{m}}$ is the point where the curve has a large change in slope (as indicated in Fig. 1(d)), giving $I_{\mathrm{m}} \sim 0.2 \mathrm{nA}$. For $|I|<I_{\mathrm{m}}\left(<I_{\mathrm{c}}\right)$ the particle in the tilted washboard potential has a high probability after thermal activation out of one potential minimum to be subsequently retrapped in the next potential minimum. The motion of the particle is therefore diffusive, leading to a small average phase velocity, i.e., a low but finite junction voltage. The branch in the $I-V_{\mathrm{J}}$ curve at $|I|<I_{\mathrm{m}}$, called the diffusive supercurrent branch, therefore has finite resistance. As the current is ramped further up $I>I_{\mathrm{m}}$ the friction in the tilted washboard potential is no longer large enough to retrap the particle once it is activated out of a minimum. This leads to a high phase velocity 

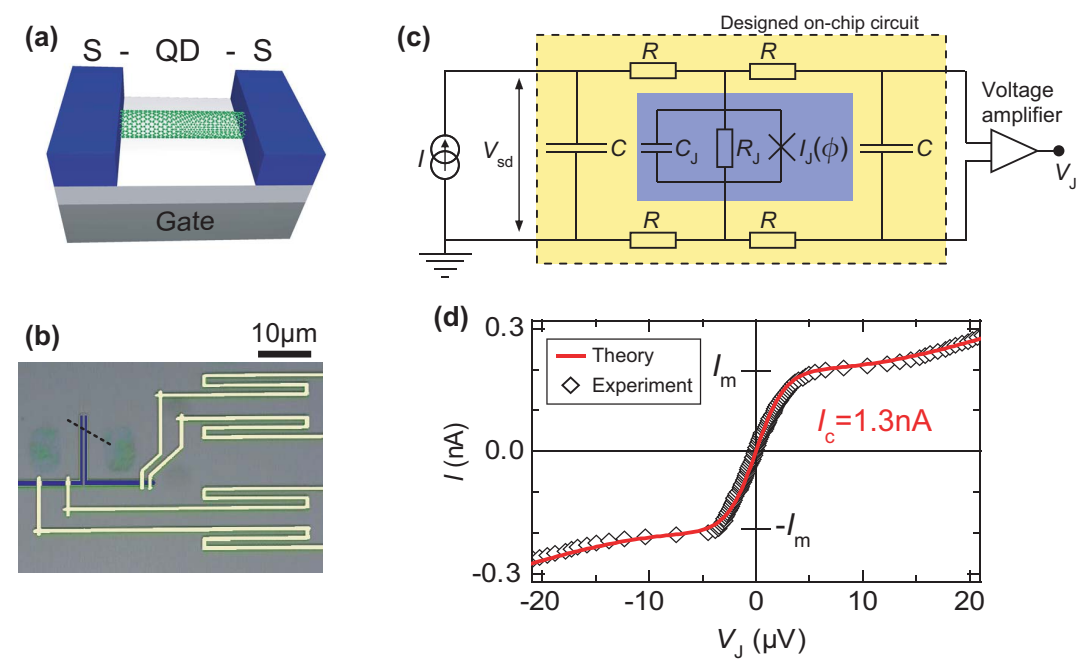

FIG. 1: Sample design and measurement scheme. (a) Schematic illustration of the Superconductor(S)-Quantum dot(QD)Superconductor(S) part of the device, made of a single wall carbon nanotube and a superconducting trilayer of $5 \mathrm{~nm}$ Ti, $60 \mathrm{~nm}$ $\mathrm{Al}$, and $5 \mathrm{~nm} \mathrm{Ti}$, with transition temperature $T_{\mathrm{c}}=0.75 \mathrm{~K}$ and energy gap $\Delta=0.1 \mathrm{meV}[5$. (b) Colored optical image of the device. Blue leads are the superconducting electrodes with a spacing of $300 \mathrm{~nm}$, contacting a nanotube (represented by a dashed line). Yellow leads are long thin $\mathrm{Cr} / \mathrm{Au}(15 \mathrm{~nm} / 15 \mathrm{~nm})$ leads connecting the superconducting leads to large-area contact pads, outside the image. (c) Schematic circuit diagram of the device with four-probe current-controlled measurement setup. Blue square: S-QD-S junction as depicted in (a). Yellow region: The resistors $R$ are the yellow leads in (b), and the capacitors $C$ are large-area contact pads. (d) Current $(I)$ versus junction voltage $\left(V_{\mathrm{J}}\right)$ close to a resonance (see the corresponding arrow in Fig. 4(b)). Measurement (diamonds) are fitted with Eq. 22 (red line) yielding a critical current $I_{\mathrm{c}}=1.3 \mathrm{nA}$ and a junction resistance $R_{\mathrm{J}}=90 \mathrm{k} \Omega$.
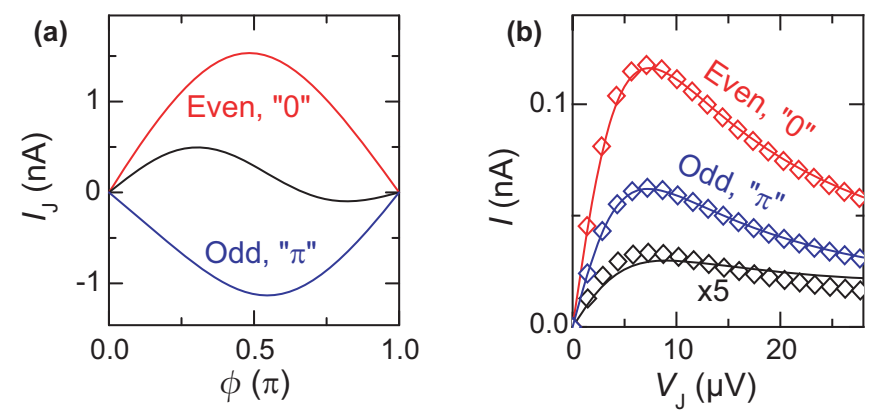

FIG. 2: Fitting procedure for extracting the critical current. (a) Theoretical current-phase relations calculated using a phenomenological "external exchange" model [17, with $E_{\text {ex }} \equiv U / 2=15 \Delta, \Gamma=\Gamma_{\mathrm{L}}+\Gamma_{\mathrm{R}}=11 \Delta, \Gamma_{\mathrm{L}}=\Gamma_{\mathrm{R}}, T=$ $\Delta / 10, I_{0}=e \Delta / \hbar \simeq 25 \mathrm{nA}$. The three curves are calculated very close to resonance at $\Delta \varepsilon=U / 30,-U / 60,-U / 15$ from top to bottom (see arrows in Fig. 4 (a)), where $\Delta \varepsilon$ is the potential on the quantum dot measured from resonance. Even though this model is phenomenological it seems to capture correctly all qualitative features of the $0-\pi$ transition known from the numerical renormalization group 24] or quantum Monte Carlo 25] calculations. Diamonds in (b) are $I-V_{\mathrm{J}}$ curves calculated using the current-phase relations from (a) in the full theory 26, valid for a general current-phase relation and in the presence of thermal fluctuations. Eq. (2) is fitted to the diamonds (solid lines) yielding very good fits in the whole gate voltage range apart from the closest vicinity of the $0-\pi$ transitions, which is below the experimental resolution. (See also the Supporting Information, Section S2).

and hence the supercurrent is averaged out.

In order to find $I_{\mathrm{c}}$, we fit the measured $I-V_{\mathrm{J}}$ curves to an overdamped $(Q<1)$ extended RCSJ model. For the overdamped Josephson junction we need to consider the classical dynamics only. In Ref. 27] the overdamped RCSJ model (without $R_{J}$ ) was calculated for sinusoidal current-phase relation and was extended to general current-phase relations in Ref. 26. High-resistance tunnel junctions have sinusoidal current-phase relations, but QD junctions with resistances comparable to the resistance quantum $h / e^{2}$ may have non-sinusoidal currentphase relation (see, e.g., Eq. (2) in Ref. [5]). Nevertheless, we approximate the current-phase relation by the simple sinusoidal form parameterized by $I_{\mathrm{c}}$, and we justify this approximation in Fig. 2 by comparing with a theoretical calculation where the full non-sinusoidal current-phase relation is included (see also the Supporting Information, Section S2). We generalize the theory of Ref. [27] to include current carried via multiple Andreev reflections by the resistor $R_{\mathrm{J}}$ which is assumed to be much larger than the lead resistance $R$. The subsequent fitted values of $R_{\mathrm{J}}$ are consistent with this assumption, see Fig. 4(c). Under these assumptions $\left(I_{\mathrm{J}}(\phi)=I_{\mathrm{c}} \sin \phi\right.$ and $\left.R_{\mathrm{J}} \gg R\right)$ the $I-V_{\mathrm{J}}$ curve is given parametrically via $V_{\mathrm{sd}}$ by the relations

$$
\begin{aligned}
& I\left(V_{\mathrm{sd}}\right)=I_{\mathrm{c}} \operatorname{Im}\left[\frac{I_{1-i \eta\left(V_{\mathrm{sd}}\right)}\left(I_{\mathrm{c}} \hbar / 2 e k_{B} T\right)}{I_{-i \eta\left(V_{\mathrm{sd}}\right)}\left(I_{\mathrm{c}} \hbar / 2 e k_{B} T\right)}\right]+\frac{V_{\mathrm{J}}\left(V_{\mathrm{sd}}\right)}{R_{\mathrm{J}}}, \\
& V_{\mathrm{J}}\left(V_{\mathrm{sd}}\right)=V_{\mathrm{sd}}-R I\left(V_{\mathrm{sd}}\right),
\end{aligned}
$$

where $I_{\alpha}(x)$ is the modified Bessel function of the complex order $\alpha$, and $\eta\left(V_{\mathrm{sd}}\right)=\hbar V_{\mathrm{sd}} / 2 e R k_{B} T$. Since all the parameters entering Eq. (2) apart from $I_{\mathrm{c}}$ and $R_{\mathrm{J}}$ are experimentally known we can use $I-V_{\mathrm{J}}$ curves to determine both $I_{\mathrm{c}}$ and $R_{\mathrm{J}}$. The solid curve in Fig. 1 (c) is a fit of Eq. (2) to the measured data, yielding $I_{\mathrm{c}}=1.3 \mathrm{nA}$ and $R_{\mathrm{J}}=90 \mathrm{k} \Omega$. The theory seems to capture the experimental measurement very well, which shows that the 

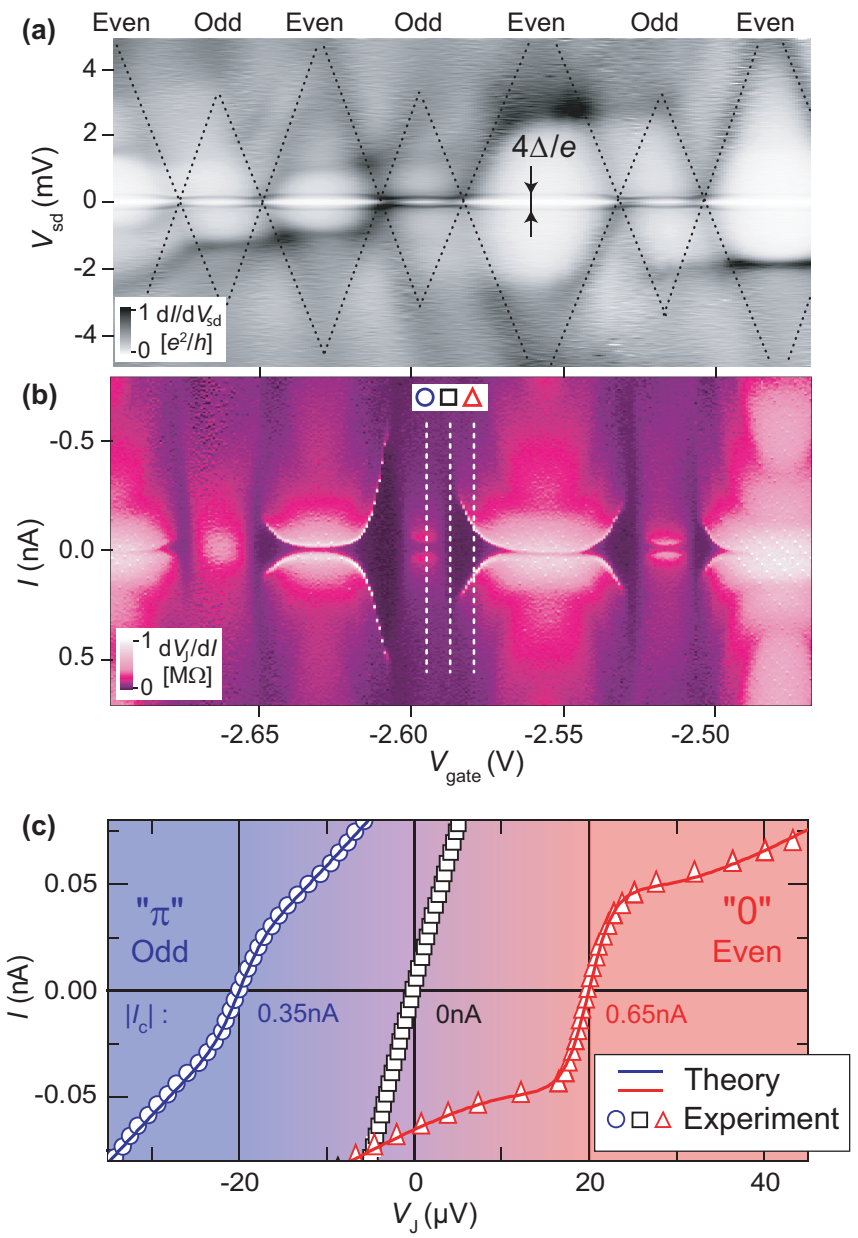

FIG. 3: Supercurrent versus odd and even electron occupation. (a) Surface plot of the differential conductance $\left(\mathrm{d} I / \mathrm{d} V_{\mathrm{sd}}\right)$ versus bias voltage $\left(V_{\mathrm{sd}}\right)$ and gate voltage $\left(V_{\text {gate }}\right)$. Coulomb blockade diamonds, indicated with black dotted lines, are alternating in size between large and small with a corresponding even and odd number of electrons localized on the QD. (b) Surface plot of differential resistance $\left(\mathrm{d} V_{\mathrm{J}} / \mathrm{d} I\right)$ versus applied current $(I)$ and $V_{\text {gate }}$, in the same gate-voltage range as in (a). (c) Three $I-V_{\mathrm{J}}$ curves from (b) at indicated positions, the right and left graphs are shifted by $20 \mu V$ for clarity. Circles are measured with odd occupation on the dot in the $\pi$ junction regime, squares at the $0-\pi$ transition point, and triangles with even occupation on the dot in the 0 junction regime. The solid lines are fits using Eq. (2) yielding critical currents of $0.65 \mathrm{nA}(0.35 \mathrm{nA})$ for even(odd) electron occupation, and zero at the transition point.

suppression of $I_{\mathrm{c}}=1.3 \mathrm{nA}$ into the diffusive supercurrent branch with $I_{\mathrm{m}}=0.2 \mathrm{nA}$ indeed is caused by thermal fluctuations. A total of five samples were fabricated with different contact resistances. They all showed $I-V_{\mathrm{J}}$ curves with qualitatively the same behavior as the sample in Fig. 1.(c) and showed excellent fits to Eq. (2) (see Supporting Information, Section S1).

In Fig. 3(a) we show a color-scale plot of the differential conductance $\left(\mathrm{d} I / \mathrm{d} V_{\text {sd }}\right)$ versus $V_{\text {sd }}$ and $V_{\text {gate }}$. Coulomb blockade diamonds, indicated with black dotted lines, alternate in size between large and small as $V_{\text {gate }}$ is increased. This indicates that the QD has two-fold spin degeneracy of each discrete energy level, with an odd (even) number of electrons on the QD in the small (large) diamond. Coulomb repulsion energy $U \sim 3 \mathrm{meV}$ and level spacing $\Delta E \sim 2.5 \mathrm{meV}$ are extracted from this plot. The edges of the Coulomb diamonds are somewhat blurred due to the tunnel coupling between QD and leads. The tunnel coupling can vary from device to device and in the Supporting Information, Section S1, we show measurements on another device (fabricated the same way) exhibiting sharer edges of the diamonds due to a slightly
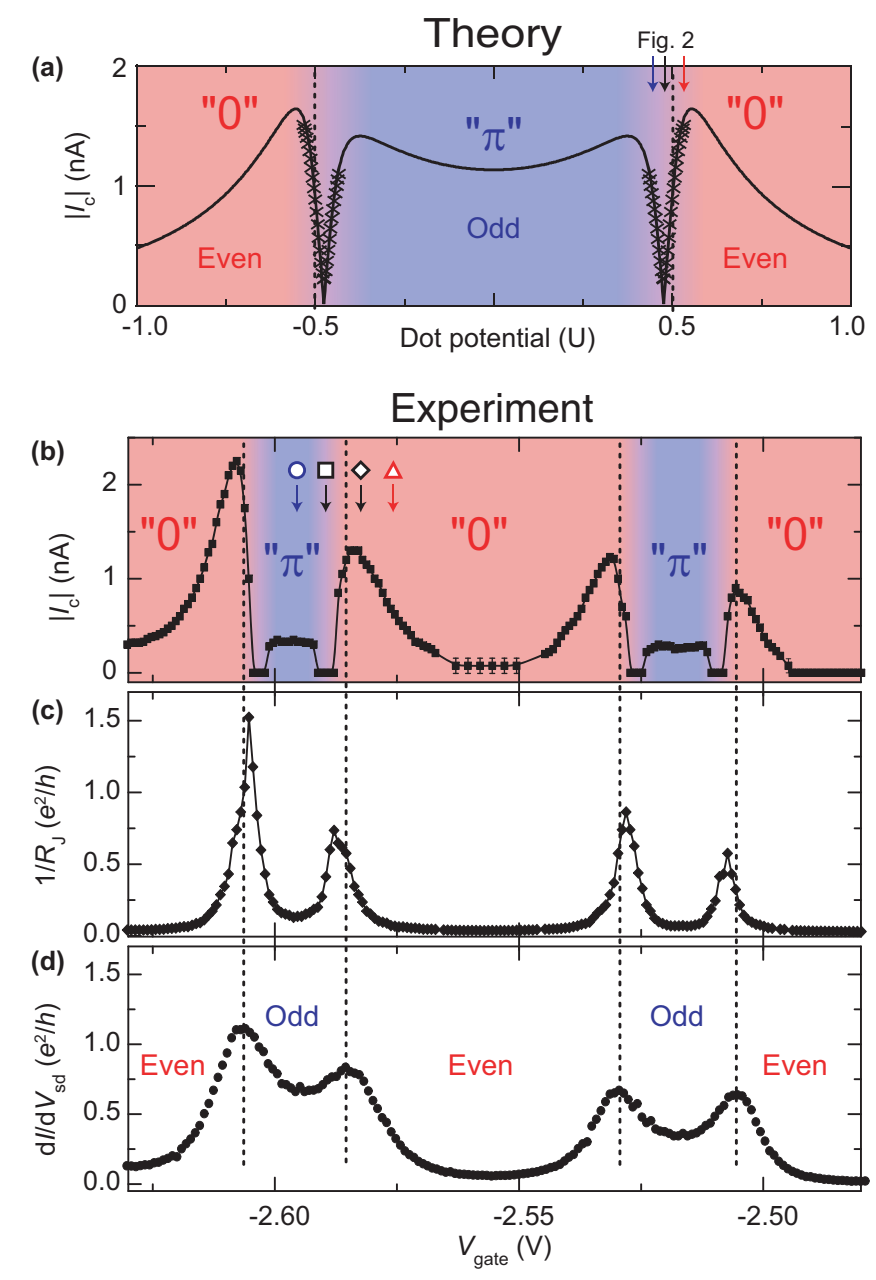

FIG. 4: Critical current $\mathbf{0}-\boldsymbol{\pi}$ transition compared to theory. (a) Solid line is $\left|I_{\mathrm{C}}\right|$ versus potential on the quantum dot obtained as $\left|I_{\mathrm{J}}(\pi / 2)\right|$ from [17] with the same parameters as in Fig. 2 Crosses are the critical currents obtained by fitting Eq. (2) to the full theory as described in Fig. 2(b). Apart from very close to the transition point, the match between the two approaches is very good. (b) and (c) Experimental critical current $\left|I_{\mathrm{C}}\right|$ and junction conductance $1 / R_{\mathrm{J}}$ as extracted from fitting measured $I-V_{\mathrm{J}}$ curves to Eq. (2), versus the gate voltage $V_{\text {gate }}$. The error-bars in (b) at the plateau around $V_{\text {gate }}=-2.56 \mathrm{~V}$ show the estimated precision of the fit. (d) Normal state differential conductance $(B=150 \mathrm{mT})$ at zero bias, in the same gate-voltage range as in (b) and (c). The dotted vertical lines indicate the positions of charge-degeneracy resonances. Note the good correspondence between the phenomenological model in (a) and the measurement in (b), both the observed magnitude of the critical current as well as the shift toward odd occupation of all the $0-\pi$ transition points are in agreement with the theory. 
lower tunnel coupling. At low bias voltage two parallel conductance ridges (indicated with black arrows) are seen, reflecting the peak in density of states of the superconductors at $V_{\mathrm{sd}}= \pm 2 \Delta / e$, yielding $\Delta \sim 0.1 \mathrm{meV}$. The energy broadening $\Gamma=\Gamma_{L}+\Gamma_{R}$, where $\Gamma_{L(R)}$ is the coupling to the left (right) lead, and the asymmetry $\Gamma_{L} / \Gamma_{R}$ of each Coulomb oscillation are determined in the normal state $(B=150 \mathrm{mT})$ by fitting the even valley part of the Coulomb oscillation peaks, Fig. 4(d), to a Lorentzian (see Supporting Information, Section S3). This gives approximately constant values of $\Gamma \sim 1.1 \mathrm{meV}$ and asymmetry parameters $\Gamma_{L} / \Gamma_{R}$ in the range 1 to 4 . These values are used in the theoretical plots in Fig. 2 and Fig. 4 (a).

In Fig. 3(b) we plot the differential resistance $\mathrm{d} V_{\mathrm{J}} / \mathrm{d} I$ as a function of $I$ and $V_{\text {gate }}$. Three representative $I-V_{\mathrm{J}}$ curves selected from Fig. 3(b) at indicated positions are shown in Fig. 3(c). The diffusive supercurrent branch is observed at most gate-voltages also at the resonances, but not in a narrow gate-region close to each resonance (open square in Fig. 3(c) and Fig.4(b)). Full gate-voltage dependence of $I_{\mathrm{c}}$, and $R_{\mathrm{J}}$ obtained by fitting the $I-V_{\mathrm{J}}$ curves from Fig. 3(b) to Eq. (2) is shown in Fig. 4(b) and (c), together with the normal-state $(B=150 \mathrm{mT})$ zero-bias conductance in Fig. $4(\mathrm{~d})$. We observe that $I_{\mathrm{c}}$ oscillates in accordance with the number of electrons (odd or even) localized on the QD. $I_{\mathrm{c}}$ furthermore exhibits sharp dips to zero for every electron added to the QD, signifying a reversal of the sign of $I_{\mathrm{c}}$ due to a 0- $\pi$ transition of the current-phase relation (see Fig. 2). All the transition points are seen to systematically shift toward odd occupation on the QD. In Fig. 4(a) we have used the phenomenological model of Ref. [17] with the same parameters as used in Fig. 2 to calculate the full gatedependence of the critical current (see Supporting Information, Section S2). Even though the QD in reality has an unpolarized magnetic moment in the odd valleys, the theory, which is based on a polarized magnetization, does capture two important consequences of the magnetic moment: (i) the magnitude of the critical current and thus its strong suppression as compared to a non-interacting resonant level $\left(I_{0}=e \Delta / \hbar \simeq 25 \mathrm{nA}\right)$, which is a result of the transport of Cooper-pairs through a strongly correlated electronic system. (ii) the slight shift of the transition points toward odd occupation.

We end by noting that Josephson junctions fabricated in $\mathrm{Al}-\mathrm{AlO}_{\mathrm{x}}-\mathrm{Al}$ are already used in superconducting quantum bits (qubits) [20, 21, 22]. The realization of a wellcontrolled Josephson QD junction in a SWCNT, opens the route towards new superconducting qubits where better control of the Josephson junctions can be achieved.

* Electronic address: hij@fys.ku.dk

[1] Kasumov, A. Y., Deblock, R., Kociak, M., Reulet, B., Bouchiat, H., Khodos, I. I., Gorbatov, Y. B., Volkov, V. T., Journet, C., and Burghard, M. Supercurrents Through Single-Walled Carbon Nanotubes. Science 284, 1508 (1999).

[2] Doh, Y.-J., van Dam, J. A., Roest, A. L., Bakkers, E. P. A. M., and Kouwenhoven, L. P. Tunable Supercurrent
Through Semiconductor Nanowires. Science 309(5732), 272-275 (2005).

[3] Takesue, I., Haruyama, J., Kobayashi, N., Chiashi, S., Maruyama, S., Sugai, T., and Shinohara, H. Superconductivity in Entirely End-Bonded Multiwalled Carbon Nanotubes. Phys. Rev. Lett. 96(5), 057001 (2006).

[4] Jarillo-Herrero, P., van Dam, J. A., and Kouwenhoven, L. P. Quantum supercurrent transistor in carbon nanotubes. Nature 436, 953-956 (2006).

[5] Jørgensen, H. I., Grove-Rasmussen, K., Novotný, T., Flensberg, K., and Lindelof, P. E. Electron Transport in Single-Wall Carbon Nanotube Weak Links in the FabryPerot Regime. Phys. Rev. Lett. 96(20), 207003 (2006).

[6] Cleuziou, J.-P., Wernsdorfer, W., Bouchiat, V., Ondaruhu, T., and Monthioux, M. Carbon nanotube superconducting quantum interference device. Nat. Nanotech. 1, 53-59 (2006).

[7] Grove-Rasmussen, K., Jørgensen, H. I., and Lindelof, P. E. Kondo resonance enhanced supercurrent in single wall carbon nanotube Josephson junctions. New. J. Phys. 9, 124 (2007).

[8] van Dam, J. A., Nazarov, Y. V., Bakkers, E. P. A. M., Franceschi, S. D., and Kouwenhoven, L. P. Supercurrent reversal in quantum dots. Nature 442, 667-670 (2006).

[9] Steinbach, A., Joyez, P., Cottet, A., Esteve, D., Devoret, M. H., Huber, M. E., and Martinis, J. M. Direct Measurement of the Josephson Supercurrent in an Ultrasmall Josephson Junction. Phys. Rev. Lett. 87(13), 137003 (2001).

[10] Joyez, P. The single Cooper pair transistor: a macroscopic quantum device. $\mathrm{PhD}$ thesis, Universit Paris 6, (1995).

[11] Ryazanov, V. V., Oboznov, V. A., Rusanov, A. Y., Veretennikov, A. V., Golubov, A. A., and Aarts, J. Coupling of Two Superconductors through a Ferromagnet: Evidence for a $\pi$ Junction. Phys. Rev. Lett. 86, 24272430 (2001).

[12] Baselmans, J. J. A., Morpurgo, A. F., van Wees, B. J., and Klapwijk, T. M. Reversing the direction of the supercurrent in a controllable Josephson junction. Nature 397, 43-45 (1999).

[13] Shiba, H. and Soda, T. Superconducting Tunneling through the Barrier with Paramagnetic Impurities. Prog. Theor. Phys. 41, 25-44 (1969).

[14] Glazman, L. I. and Matveev, K. A. Resonant Josephson current through Kondo impurities in a tunnel barrier. JETP Lett. 49, 659-662 (1989).

[15] Clerk, A. A. and Ambegaokar, V. Loss of $\pi$-junction behavior in an interacting impurity Josephson junction. Phys. Rev. B 61, 9109-9112 (2000).

[16] Spivak, B. I. and Kivelson, S. A. Negative local superfluid densities: The difference between dirty superconductors and dirty Bose liquids. Phys. Rev. B 43, 3740-3743 (1991).

[17] Vecino, E., Martín-Rodero, A., and Yeyati, A. L. Josephson current through a correlated quantum level: Andreev states and $\pi$ junction behavior. Phys. Rev. B 68(3), 035105 (2003).

[18] Ishizaka, S., Sone, J., and Ando, T. dc Josephson current through a quantum dot coupled with superconducting leads. Phys. Rev. B 52, 8358-8362 (1995).

[19] Rozhkov, A. V., Arovas, D. P., and Guinea, F. Josephson coupling through a quantum dot. Phys. Rev. B 64(23), 233301 (2001).

[20] Nakamura, Y., Pashkin, Y. A., and Tsai, J. S. Coherent control of macroscopic quantum states in a singleCooper-pair box. Nature 398, 786-788 (1999).

[21] Vion, D., Aassime, A., Cottet, A., Joyez, P., Pothier, H., Urbina, C., Esteve, D., and Devoret, M. H. Manipulating 
the Quantum State of an Electrical Circuit. Science 296, 886-889 (2002).

[22] Chiorescu, I., Nakamura, Y., Harmans, C. J. P. M., and Mooij, J. E. Coherent Quantum Dynamics of superconducting Flux Qubit. Science 299, 1869-1871 (2003).

[23] Tinkham, M. Introduction to superconductivity, chapter 6. McGraw-Hill Inc., ISBN, 0-07-064878-6 (1996).

[24] Choi, M.-S., Lee, M., Kang, K., and Belzig, W. Kondo effect and Josephson current through a quantum dot between two superconductors. Phys. Rev. B $\mathbf{7 0}(2), 020502$ (2004).

[25] Siano, F. and Egger, R. Josephson Current through a Nanoscale Magnetic Quantum Dot. Phys. Rev. Lett. 93(4), 047002 (2004).

[26] Ambegaokar, V. and Halperin, B. I. Voltage Due to Thermal Noise in the dc Josephson Effect. Phys. Rev. Lett. 22, 1364-1366 (1969).

[27] Ivanchenko, Y. M. and Zil'Berman, L. A. The Josephson Effect in Small Tunnel Contacts. JETP Lett. 28, 1272 (1969).

\section{Acknowledgements}

We acknowledge fruitful discussions with M.-S. Choi, M. H. Devoret, T. Heikkila, V. Meden, J. Paaske, C. Urbina, and W. Wernsdorfer. We thank F. B. Rasmussen for technical help. The work was in part supported by the EU-STREP ULTRA-1D and CARDEQ program, and (T. N.) by the research plan MSM 0021620834 financed by the Ministry of Education of the Czech Republic.

\section{Supporting information available}

The three sections in the Supporting Information include: (i) To demonstrate reproducibility we show similar measurements on another device. (ii) Justification of the fitting procedure and explanation of the simple model prediction for the supercurrent. (iii) Estimating the asymmetry and level broadening of the device from the Coulomb blockade peaks. This material is available free of charge via the Internet at http://pubs.acs.org 